\title{
Gastric Cancer Screening Methods: A Comparative Study of Two Scoring Methods
}

\author{
Weixing Zhang' \\ Zhangzhi $\mathrm{Li}^{2}$ \\ Muhammad Safwan Akram \\ Muhammad Fayyaz Ur \\ Rehman (ID) 4 \\ Nazeer Hussain Khan ${ }^{5,6}$ \\ Dan $\mathrm{Hu}^{7}$ \\ Muhammad Mustaqeem ${ }^{8}$ \\ Yuanyuan Zeng' \\ Fariha Kanwal (iD) \\ 'Department of Critical Care Medicine, \\ Shanghai General Hospital, Shanghai Jiao \\ Tong University, School of Medicine, \\ Shanghai, People's Republic of China; \\ ${ }^{2}$ Department of Hematology, Taihe \\ Hospital, Hubei University of Medicine, \\ Hubei, 442000, People's Republic of \\ China; ${ }^{3}$ School of Health and Life \\ Sciences, Teesside University, \\ Middlesbrough, TSI 3BX, UK; ${ }^{4}$ Institute \\ of Chemistry, University of Sargodha, \\ Sargodha, 40100, Pakistan; ${ }^{5}$ Henan \\ International Key Laboratory of Nuclear \\ Protein, School of Life Sciences, Henan \\ University Kaifeng, Henan, 475004, \\ People's Republic of China; 'Laboratory \\ of Animal and Human Physiology, \\ Department of Biological Sciences, Quaid \\ I Azam University, Islamabad, 45320, \\ Pakistan; ${ }^{7}$ Department of Neurology, The \\ Central Hospital of Xiaogan, Hubei, \\ 432100, People's Republic of China; \\ ${ }^{8}$ Department of Chemistry, University of \\ Sargodha, Sub Campus Bhakkar, Bhakkar, \\ 30000, Pakistan; ${ }^{9}$ Med-X Research \\ Institute, School of Biomedical \\ Engineering, Shanghai Jiao Tong \\ University, Shanghai, People's Republic of \\ China
}

Correspondence: Fariha Kanwal; Yuanyuan Zeng

Email fariha@sjtu.edu.cn;

yuanyuanzeng@aliyun.com
Objective: To evaluate the Li's and Japanese scoring methods scoring for screening early gastric cancer in a healthy population.

Methods: During January 2016-December 2018, profiles of the healthy people participated in a physical examination in the first people's Hospital of Shanghai were collected. A total of 342 volunteers, including 137 males and 205 females ageing 40-74, were enrolled. After recording the basic information, all volunteers were scored using the Japan scoring method and the new gastric cancer screening score (ie, Li's score). The subjects' work characteristics (ROC curve) were drawn according to the patient's endoscopic pathological examination to indicate early gastric cancer, to determine the best cut-off point for the diagnosis of early gastric cancer by Japanese scoring and Li's scoring, respectively. The sensitivity and specificity of both scoring methods were calculated as well.

Results: The area under the ROC curve of Japanese and Li's score, in the diagnosis of early gastric cancer, was 0.763 and 0.837 , respectively. Japanese and Li's score $\geq 14$ were considered as the best cut-off point. The sensitivity and specificity of Li's scoring were $63.60 \%$ and $91.10 \%$, respectively. The sensitivity and specificity of the Japanese score were $54.50 \%$ and $87.50 \%$, respectively. The area under the ROC curve in Li's scoring is more significant than that in Japanese scoring, and there was a substantial difference in the two methods $(\mathrm{P}<0.05)$.

Conclusion: Both Li's scoring and Japanese scoring have shown good screening value for early gastric cancer in a healthy population, but Li's scoring is more sensitive/specific than Japanese scoring.

Keywords: Li's scoring, Japanese scoring, early gastric cancer

\section{Introduction}

Gastric cancer is the sixth most common cancer in the world and the second most common cancer leading to death. ${ }^{1}$ There are huge differences in the global distribution of morbidity and mortality associated with gastric cancer, with the highest incidence in East Asia. ${ }^{2}$ The incidence and mortality of gastric cancer in China has reached an alarming level with $42.6 \%$ and $45.0 \%$, respectively, while the number of new cases continues to increase due to population growth and population ageing. Gastric cancer affects men and women disproportionately, and the incidence of men is almost two times higher than that of women. ${ }^{3}$ A global survey indicated that the incidence of gastric cancer was $35.4 \%$ in men and $13.8 \%$ in women. ${ }^{4}$ Despite improvements in the diagnosis and treatment of gastric cancer, the mortality rate of this cancer is still very high. The 5-year survival rate of gastric cancer in most countries in the world is only $20 \%{ }^{5}$ Japan is one of the ten countries with the highest prevalence of gastric cancer in 
the world, and has made an important contribution to better understanding the pathogenesis of gastric cancer and early endoscopic screening to reduce its prevalence. ${ }^{6}$ In Japan, a $50 \%$ reduction in gastric cancer-related mortality has been observed between 1975 and 2005. ${ }^{2}$ Early diagnosis of gastric cancer and better control of the risk factors can reduce the global incidence of gastric cancer.

At present, about $90 \%$ of gastric cancer found in China belong to the progressive stage, while the prognosis of gastric cancer is closely related to the timing of diagnosis and treatment, and the 5-year survival rate after the treatment of early gastric cancer can exceed $90 \%$, and even achieve the cure effect. ${ }^{7}$ However, the rate of diagnosis and treatment of early gastric cancer in China is less than $10 \%$, which is much lower than that in Japan $(70 \%)$ and Korea $(50 \%) .{ }^{8}$ In the Chinese Three-Year Action Plan for Cancer Prevention and Control (2015-2017), it has been clearly stated that there is a need to promote and improve cancer screening, early diagnosis and early treatment strategies.

A total of 19,028 subjects were enrolled in the follow-up visit by the Japanese public health center between 1993 and 2009. Based on age, living habits (smoking, high salt), family history of gastric cancer and $\mathrm{ABC}$ method grouping data, a Japanese scoring method for gastric cancer screening in the population was established. ${ }^{9}$ However, whether the Japanese scoring model is applicable to the Chinese population remains elusive. The National Center for Clinical Medicine Research of Digestive Diseases (Shanghai) has recently carried out a big data, multi-center clinical study involving more than 120 hospitals in China. Based on this study, a new gastric cancer screening scoring system has been established. The purpose of this study is to preliminarily verify the value of the Japanese scoring and propose a new scoring method, ie the Li-Q score, for gastric cancer prediction in the early cancer stages by screening healthy people, and to compare the accuracy between the two scoring methods.

\section{Materials and Methods}

\section{General Information}

This study was a single-center, retrospective study. The healthy people who participated in the physical examination in the first people's hospital of shanghai during January 2016December 2018 were enrolled. Participation criteria include: (1) age 40-80 years, both male and female are included; (2) willing to be screened and be able to cooperate with the completion. Exclusion criteria were as follows: (1) persons taking drugs (including proton pump inhibitors, $\mathrm{H}$ receptor antagonists, and gastric mucosal protectants), within 2 weeks since participating physical examination that could affect the observation in this study; (2) having severe cardiac, liver, and renal insufficiency, severe neuropathy, or mental disorders; and (3) having a tendency to bleed and cannot undergo a biopsy. This study has been approved by the Ethics Committee of Shanghai First People's Hospital and the consent of the subjects has been obtained. All participants were informed about the purpose of the study, and that it was conducted in accordance with the Declaration of Helsinki.

\section{Japanese Scoring and New Gastric Cancer Screening Scores}

The Japanese public health center has followed up 19,028 subjects between 1993 and 2009 and found 412 gastric cancer cases in 270,854 persons in these years (average 14.2 years). Based on this data, a prediction model of gastric cancer risk was established. ${ }^{9}$ This model was scored on the basis of age, lifestyle (smoking, high salt), family history of gastric cancer, and ABC method grouping data. According to the score, the screening population was divided into 3 grades, 0-14 were divided into the lowrisk group, 15 19 were divided into the medium risk group, and 20-23 were divided into the high-risk group (Table 1).

\section{New Gastric Cancer Screening Scoring System}

A new gastric cancer screening scoring system has been proposed in the expert consensus opinion of the early

Table I Novel Scoring System for Gastric Cancer Screening

\begin{tabular}{|c|c|c|}
\hline Variates & Classification & Score \\
\hline \multirow{4}{*}{ Age (years) } & $40 \sim 49$ & 0 \\
\hline & $50 \sim 9$ & 5 \\
\hline & $60 \sim 69$ & 6 \\
\hline & $>69$ & 10 \\
\hline \multirow{2}{*}{ Gender } & Female & 0 \\
\hline & Male & 4 \\
\hline \multirow[t]{2}{*}{ Helicobacter pylori antibody } & Negative & 0 \\
\hline & Positive & 1 \\
\hline \multirow[t]{3}{*}{ Serum pepsinogen $1 / I$ ratio } & $\geq 3.89$ & 0 \\
\hline & $<3.89$ & 3 \\
\hline & $<1.50$ & 0 \\
\hline \multirow[t]{2}{*}{ Gastrin 17 (pmol/L) } & $1.50 \sim 5.70$ & 3 \\
\hline & $>5.70$ & 5 \\
\hline
\end{tabular}


gastric cancer screening process in China. ${ }^{10-12}$ It included 5 variables with a total score of 23: (1) Age: 0 for 40-49, 5 for 50-59, 6 for 60-69, and 10 for $>69$; (2) Sex: 4 for male and 0 for female; (3) Helicobacter pylori infection (Hp antibody test): positive 1 , negative 0 ; (4) PGR: $\geq 3.89$ is 0 , $<3.89$ is 3 ; (5) G-17: less than 1.50 is $0,1.50-5.70$ is 3 , greater than 5.70 is 5 . The screening population was divided into 3 grades according to the score, 0-11 into low-risk group, 12-16 into medium risk group and 17-23 into high-risk group (Table 2).

Demographic information, smoking history, consumption of pickled foods, and family history of gastric cancer, breath test, Helicobacter pylori antibody, and serum pepsinogen detection results were recorded after hospital registration. The risk of gastric cancer was assessed according to the combination of serum PG and H. pylori antibodies (i.e.," $\mathrm{ABC}$ method"). All the volunteers were then given a Japanese model score and a new gastric cancer screening score, and the subjects' work characteristics (receiver operator characteristic, ROC) were plotted on the basis of endoscopic pathology indicating early gastric cancer curve to determine the best cutoff point of the Japanese model score and the new gastric cancer screening score in the diagnosis of early gastric cancer, to analyze their diagnostic value, and to compare the accuracy between the two scoring methods.

\section{Statistical Treatment}

SPSS ver. 22.0 software was used for the statistical analysis. Pathology results were regarded as the golden standard. ROC curve was used to determine the best cut-off point

Table 2 Simplified Scoring Sheet for Risk Assessment of Gastric Cancer in 10 Years

\begin{tabular}{|c|c|c|c|}
\hline Items & Score & Items & Score \\
\hline Age (year) & & Family history & \\
\hline $40-44$ & Female 0 Male I & No & 0 \\
\hline $45-49$ & Female I Male 3 & Yes & I \\
\hline $50-54$ & Female 2 Male 4 & $\begin{array}{l}\text { ABC risk } \\
\text { stratification }\end{array}$ & \\
\hline $55-59$ & Female 3 Male 6 & A & 0 \\
\hline $60-64$ & Female 4 Male 8 & B & 8 \\
\hline $65-71$ & $\begin{array}{l}\text { Female } 5 \text { Male } \\
10\end{array}$ & C & II \\
\hline Smoking & & $\mathrm{D}$ & II \\
\hline Never before & 0 & & \\
\hline Smoking now & I & Total score & $0-24$ \\
\hline \multicolumn{4}{|l|}{ Eating salted caviar } \\
\hline No & 0 & & \\
\hline Yes & I & & \\
\hline
\end{tabular}

for diagnosis of early gastric cancer, and the sensitivity and specificity were calculated. The $\mathrm{Z}$ test was also used to compare the area under the ROC curve (accuracy comparison) for the two scores. $\mathrm{P}<0.05$ was statistically significant.

\section{Results}

\section{General Situation}

Due to the contraindication of gastroscopy and the poor tolerance of the population to gastroscopy, a large proportion of people are unwilling to undergo routine gastroscopy screening, a total of 342 volunteers were included (Table 3), 137 males and 205 females, aged 40-74 years. The proportion of people over 60 years of age was the largest, 185 (54.1\%), and $13.4 \%$ had a history of smoking, $68.7 \%$ had eaten pickled foods, $11.3 \%$ had a family history of gastric cancer, $44.2 \%$ had positive breath tests, and $26.9 \%$ had Helicobacter pylori antibodies.

\section{Comparison of Risk Stratification Results of Two Scoring Methods}

Early gastric cancer risk stratification in 342 subjects: 313 low-risk cases (91.52\%), 27 medium risk cases (7.89\%) and 2 high-risk cases $(0.58 \%)$ of early gastric cancer were diagnosed by Japanese scoring. The diagnose rate of Li's scoring of low-risk cases, medium risk cases and high-risk cases were $(77.19 \%, 264),(21.93 \%, 75)$, and $(0.88 \%, 3)$, respectively (Table 4).

Table 3 General Information of Participants $(\mathrm{N}=342)$

\begin{tabular}{|l|l|}
\hline Age (year) & $\mathbf{4 0 - 7 4}$ \\
\hline Male/Female (case) & $137: 205$ \\
\hline $\begin{array}{l}\text { Age (\%) } \\
40-50[\text { case number (\%)] } \\
5 \mathrm{I}-60 \text { [case number (\%)] } \\
>60[\text { case number (\%)] }\end{array}$ & $\begin{array}{l}53(15.5) \\
104(30.4) \\
185(54.1)\end{array}$ \\
\hline $\begin{array}{l}\text { Smoking history } \\
\text { Yes [case number (\%)] }\end{array}$ & $46(13.4)$ \\
\hline $\begin{array}{l}\text { Pickled food eating } \\
\text { Yes [case number (\%)] }\end{array}$ & $235(68.7)$ \\
\hline $\begin{array}{l}\text { Family history of gastric cancer } \\
\text { Yes [case number (\%)] }\end{array}$ & $33(11.3)$ \\
\hline $\begin{array}{l}\text { Exhale test } \\
\text { Positive [case number (\%)] }\end{array}$ & 151 (44.2) \\
\hline $\begin{array}{l}\text { Helicobacter pylori antibody } \\
\text { Yes [case number (\%)] }\end{array}$ & $92(26.9)$ \\
\hline
\end{tabular}


Table 4 Comparison of Risk Stratification Results Between the Two Scoring Methods (Case \%)

\begin{tabular}{|l|c|c|c|c|}
\hline \multirow{2}{*}{$\begin{array}{l}\text { Scoring } \\
\text { Method }\end{array}$} & $\begin{array}{c}\text { Case } \\
\text { Number }\end{array}$ & \multicolumn{3}{|c|}{ Risk Stratification } \\
\cline { 3 - 5 } & & Low Risk & $\begin{array}{c}\text { Medium } \\
\text { Risk }\end{array}$ & $\begin{array}{c}\text { High } \\
\text { Risk }\end{array}$ \\
\hline Japanese scoring & 342 & $313(91.52)$ & $27(7.89)$ & $2(0.58)$ \\
Li' Scoring & 342 & $264(77.19)$ & $75(21.93)$ & $3(0.88)$ \\
\hline
\end{tabular}

\section{ROC Curve Analysis}

The area under the ROC curve of the Japanese score for the diagnosis of early gastric cancer was 0.763 , and the best cut-off point was 13.5, which means the Japanese score $>13.5$ was regarded as early gastric cancer patients, and the sensitivity and specificity were $54.50 \%$ and $87.50 \%$, respectively. The area under the ROC curve of the Li's score for the diagnosis of early gastric cancer was 0.837 , and the best cut-off point was 13.5 , which means Li's score $>13.5$ was regarded as early gastric cancer patients, and the sensitivity and specificity were $63.60 \%$ and $91.10 \%$, respectively (Figure 1). It concluded that the area under the ROC curve of Li's score (0.837) was larger than that under the ROC curve of Japan's score (0.763), which has a significant difference after the $Z$ test $(p>0.01, Z=2.63$. The sensitivity and specificity of each cut-off point of Li's scoring and Japan's scoring are given in Table 5 .

\section{Comparison of Japanese and New Gastric Cancer Screening Score Accuracy}

The area under the ROC curve of Li's score (0.837) was larger than that under the ROC curve of Japan's score (0.763), which was a significant difference after the $\mathrm{Z}$ test $(\mathrm{P}<0.05)$.

\section{Discussion}

Cancer is a sort of disease that results from abnormal cell proliferation. Despite the various syndromes of diverse cancers, uncontrolled cell growth is the most common symptom. ${ }^{13,14}$ Gastric cancer is one of the most common types of cancer, but at early stages, it does not show any special signs and symptoms that makes an early diagnosis of gastric cancer very difficult. Nevertheless, scientists have identified a number of predisposing factors, including bacterial infection, ${ }^{15}$ gender difference, age, race, environment, diet, ${ }^{16}$ and history of gastric surgery, pernicious anemia, infectious diseases, and blood-type factors. ${ }^{17}$ Although the global prevalence of gastric cancer is declining, the prevalence of this disease remains high in Asian countries. ${ }^{18}$ So, early-stage screening to achieve an early treatment for gastric cancer is the focus and difficulty of medical workers.

The 5-year survival rate for gastric cancer in China is low, and there is a delayed diagnosis. ${ }^{19}$ Factors contributing to this delay may include poor understanding of risk factors or symptoms, and negative attitudes towards screening for

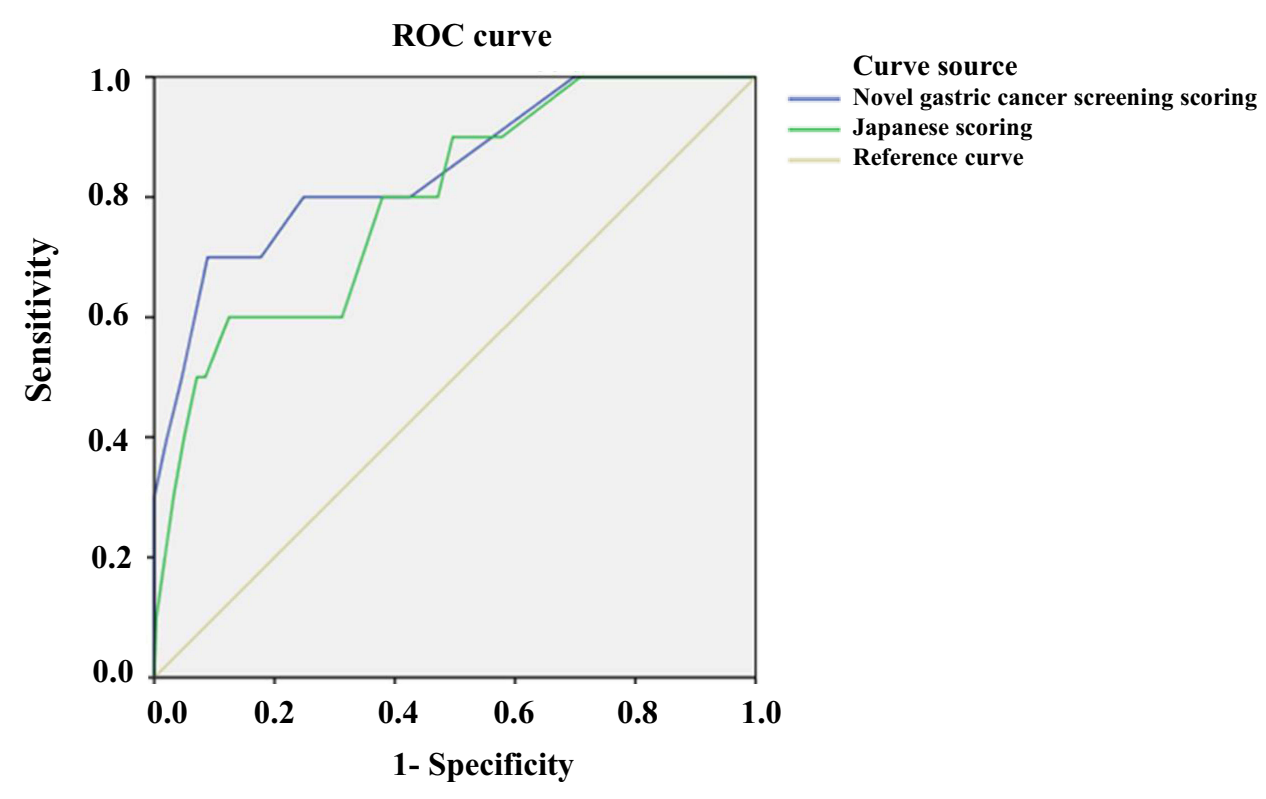

Figure I Shows the ROC curve analysis of two scoring methods to diagnose early gastric cancer. 
Table 5 Novel Scoring System for Gastric Cancer Screening

\begin{tabular}{|c|c|c|c|c|c|}
\hline \multicolumn{3}{|c|}{ Li's Scoring } & \multicolumn{3}{|c|}{ Japanese Scoring } \\
\hline Cut-Off Point & Sensitivity & Specificity & Cut-Off Point & Sensitivity & Specificity \\
\hline 9.5 & 0.818 & 0.577 & 9.5 & 0.727 & 0.619 \\
\hline 10.5 & 0.818 & 0.69 & 10.5 & 0.545 & 0.687 \\
\hline 11.5 & 0.727 & 0.751 & 11.5 & 0.545 & 0.74 \\
\hline 12.5 & 0.636 & 0.769 & 12.5 & 0.545 & 0.811 \\
\hline 13.5 & 0.636 & 0.911 & 13.5 & 0.545 & 0.875 \\
\hline 14.5 & 0.455 & 0.954 & 14.5 & 0.455 & 0.915 \\
\hline 15.5 & 0.364 & 0.979 & 15.5 & 0.455 & 0.929 \\
\hline
\end{tabular}

gastric cancer. ${ }^{20}$ Gastric cancer is a multi-stage and multifactor-related disease. At present, the most studied influencing factors are lifestyle, but there are still some differences in specific projects. ${ }^{21} \mathrm{H}$. pylori infection has been identified as the main cause of gastric cancer, and almost $90 \%$ of gastric cancer cases are attributed to H. pylori infection. ${ }^{22}$ In this study, participants who showed positive results in expiratory tests accounted for $44.2 \%$, which may be related to the eating habits of residents. In this study, $11.3 \%$ of participants had a family history of gastric cancer. A related study shows that the proportion of family gastric cancer in patients was higher than that in healthy people, which may be caused by the presence of $\mathrm{CDH} 1$ susceptible gene inheritance in patients with a family history of gastric cancer. Therefore, understanding the risk factors of gastric cancer can provide guidance for the early prevention of gastric cancer.

Gastric cancer risk score screening in a healthy population is the basis for the next step into endoscopic screening, which can effectively improve the positive detection rate of endoscopic examination. It has the characteristics of high efficiency, low cost, good compliance, simple and suitable for large-scale gastric cancer screening. Results of early gastric cancer risk stratification with the Japanese scoring method in 342 subjects showed that the cases of low risk, medium risk and high risk were 313 cases (91.52\%), 27 cases $(7.89 \%)$ and 2 cases $(0.58 \%)$, respectively.

In Li's scoring, 264 cases (77.19\%) were diagnosed as low risk, 75 cases $(21.93 \%)$ were diagnosed as medium risk and 3 cases $(0.88 \%)$ were diagnosed as high risk. By drawing the ROC curve, it was found that the best cut-off points of both Li's score and Japan's score were 13.5. Since the actual score can only be an integer, the best cutoff points of Li's score and Japan's score were $>13.5$. The sensitivity and specificity of two scoring methods were $63.60 \%, 91.10 \%$ and $54.50 \%$ and $87.50 \%$, respectively.
Regarding the comparison of the accuracy between the two scoring methods, Li's scoring method was more accurate than that of Japan.

Li's scoring method was more valuable for the diagnosis of early gastric cancer. Based on the results of statistical analysis, Li's scoring is more accurate and superior in predicting early gastric cancer. However, through a comprehensive evaluation of the two methods, it was found that either method has advantages and disadvantages, and in clinical practice, both scoring methods should be given equal attention. Carefully reviewing each item of the two scoring methods, we noticed that the index weight setting for the Japanese scoring model was debatable Reduction of serum G-17 levels could be used as diagnose index of antral atrophy. However, the G-17 level decline has not been included in the scoring model. In addition, it was inappropriate to count $\mathrm{HpAb}$ negative to 0 because this could also occur in patients with severe atrophy of gastric mucosa and extensive intestinal metaplasia.

A total of 5 variables, age, gender difference, H. pylori antibody, PG and G-17, were assigned different scores (weights) in the new gastric cancer screening scoring system. As environmental (diet and alcohol and tobacco) and genetic (family history of gastric cancer) factors were risk factors of precancerous disease in the target population of gastric cancer screening, ${ }^{23}$ the ability to distinguish gastric cancer from precancerous disease is limited. Thus, the two factors have not been integrated into the new gastric cancer screening scoring system after statistical analysis. The same principle also applied to H. pylori infections, which also belong to the traditional gastric cancer screening index of the target population, but the score is only slightly elevated (listed as 1). PG I/PG II is more significant than PG I, and is regarded as the newly added quantisation scoring item, along with G-17. The score of the two items fully reflected the risk of gastric 
cancer, together with the age and gender, constituted the basis of the new scoring system, which was different from the previous gastric cancer risk scoring system.

Understanding the risk factors for gastric cancer is an important prerequisite for healthy behavior. In addition, knowledge about warning symptoms is critical for early diagnosis of cancer. Abdominal mass, presence of abdominal fullness and pain are warning symptoms of gastric cancer. A recent study has shown that knowledge of warning symptoms can lead to earlier medical treatment, leading to earlier diagnosis and better prognosis results. ${ }^{24}$ Attitudes towards screening are significantly associated with screening behavior. ${ }^{25}$ In China, there are no national guidelines or procedures for gastric cancer screening to the date $^{20}$ In clinical practice, it is recommended that highrisk groups (eg people with H. pylori infection) should undergo gastric cancer screening. ${ }^{26}$ In China, public awareness of risk factors or warning symptoms of gastric cancer and the popularization of knowledge of gastric cancer screening should be strengthened.

This study is a single-center, retrospective study that, to some extent, affected the accuracy and reliability of the results. The main inadequacies of this study include a relatively small sample size and the lack of further follow-up of patients. In the future, a continued work on a multicenter, prospective study will be launched further to evaluate Li's scoring and Japan's scoring and to obtain a more accurate and more Chinese population applicative new method for gastric cancer screening.

\section{Conclusions}

Both Li's scoring and Japanese scoring have shown good screening value for early gastric cancer in a healthy population, but Li's scoring is more sensitive/specific than Japanese scoring.

\section{Acknowledgments}

The authors pay acknowledge this research to the National Natural Science Foundation of China (No. 81870452) and Shanghai Natural Science and Technology Fund (No.19441906700). The authors would like to express their thankfulness to the anonymous referees who helped to improve this manuscript. Hussain is deeply thankful to his Piayree Lala whose presence gives him reason and strength for this contribution in science.

\section{Author Contributions}

All authors made substantial contributions to conception and design, acquisition of data, or analysis and interpretation of data, took part in drafting the article or revising it critically for important intellectual content, agreed to submit to the current journal, gave final approval for the version to be published, and agreed to be accountable for all aspects of the work.

\section{Disclosure}

The authors declare no conflicts of interest.

\section{References}

1. Bray F, Ferlay J, Soerjomataram I, Siegel RL, Torre LA, Jemal A. Global cancer statistics 2018: GLOBOCAN estimates of incidence and mortality worldwide for 36 cancers in 185 countries. CA Cancer J Clin. 2018;68:394-424. doi:10.3322/caac.21492

2. Hamashima C. Current issues and future perspectives of gastric cancer screening. World J Gastroenterol. 2014;20:13767.

3. den Hoed CM, Kuipers EJ. Gastric cancer: how can we reduce the incidence of this disease? Curr Gastroenterol Rep. 2016;18:34. doi:10.1007/s11894-016-0506-0

4. Neugut AI, Hayek M, Howe G. Epidemiology of gastric cancer. Proce Sem Oncol. 2015:281-291.

5. Karimi P, Islami F, Anandasabapathy S, Freedman ND, Kamangar F. Gastric cancer: descriptive epidemiology, risk factors, screening, and prevention. Cancer Epidemiol Prevent Biomarkers. 2014;23:700-713. doi:10.1158/1055-9965.EPI-13-1057

6. Isobe Y, Nashimoto A, Akazawa K, et al. Gastric cancer treatment in Japan: 2008 annual report of the JGCA nationwide registry. Gastric Cancer. 2011;14:301-316. doi:10.1007/s10120011-0085-6

7. Sumiyama K. Past and current trends in endoscopic diagnosis for early stage gastric cancer in Japan. Gastric Cancer. 2017;20:20-27. doi:10.1007/s10120-016-0659-4

8. Ren W, Yu J, Zhang Z-M, Song Y-K, Li Y-H, Wang L. Missed diagnosis of early gastric cancer or high-grade intraepithelial neoplasia. World J Gastroenterol. 2013;19:2092.

9. Charvat H, Sasazuki S, Inoue M, et al. Prediction of the 10-year probability of gastric cancer occurrence in the $\mathrm{J}$ apanese population: the JPHC study cohort II. Int $j$ Cancer. 2016;138:320-331. doi: $10.1002 / \mathrm{ijc} .29705$

10. Du Y, Zhu H, Liu J, et al. Consensus on eradication of Helicobacter pylori and prevention and control of gastric cancer in China (2019, Shanghai). J Gastroenterol Hepatol. 2020;35:624-629. doi:10.1111/ jgh. 14947

11. Liu WZ, Xie Y, Lu H, et al. Fifth Chinese National Consensus Report on the management of Helicobacter pylori infection. Helicobacter. 2018;23:e12475. doi:10.1111/hel.12475

12. Zhang K-C, Chen L, Chinese A CR; Association, C.G.C.; Committee, C.R.H.A.D.T. Chinese expert consensus and practice guideline of totally implantable access port for digestive tract carcinomas. World J Gastroenterol. 2020;26(25):3517. doi:10.3748/ wjg.v26.i25.3517

13. de Castro Mayor J, Caño JV, Aragón JC, Andrés GB, Herranz FA, Hernández CF. Locally advanced prostate cancer. Definition, diagnosis and treatment. Arch Esp Urol. 2018;71:231-238.

14. Kim H-I, Park M, Song K, Woo Y, Hyung W. Rapid and safe learning of robotic gastrectomy for gastric cancer: multidimensional analysis in a comparison with laparoscopic gastrectomy. Eur $J$ Surg Oncol. 2014;40:1346-1354. doi:10.1016/j. ejso.2013.09.011 
15. Bartfeld S, Bayram T, van de Wetering M, et al. In vitro expansion of human gastric epithelial stem cells and their responses to bacterial infection. Gastroenterology. 2015;148:126-136. e126. doi:10.1053/j. gastro.2014.09.042

16. Shimada S, Sawada N, Ishiyama Y, et al. Impact of obesity on short-and long-term outcomes of laparoscopy assisted distal gastrectomy for gastric cancer. Surg Endosc. 2018;32:358-366. doi:10.1007/ s00464-017-5684-9

17. Alkebsi L, Ideno Y, Lee J-S, et al. Gastroduodenal ulcers and ABO blood group: the Japan nurses' health study (JNHS). J Epidemiol. 2018;28:34-40. doi:10.2188/jea.JE20160204

18. Zhu L, Luo W, Su M, et al. Comparison between artificial neural network and Cox regression model in predicting the survival rate of gastric cancer patients. Biomedical Rep. 2013;1:757-760. doi:10.3892/br.2013.140

19. Allemani C, Weir HK, Carreira H, et al. Global surveillance of cancer survival 1995-2009: analysis of individual data for 25676887 patients from 279 population-based registries in 67 countries (CONCORD-2). Lancet. 2015;385:977-1010. doi:10.1016/S01406736(14)62038-9

20. Zong L, Abe M, Seto Y, Ji J. The challenge of screening for early gastric cancer in China. Lancet. 2016;388:2606. doi:10.1016/S01406736(16)32226-7
21. Yoon H, Kim N. Diagnosis and management of high risk group for gastric cancer. Gut Liver. 2015;9:5. doi:10.5009/gnl14118

22. Plummer M, Franceschi S, Vignat J, Forman D, de Martel C. Global burden of gastric cancer attributable to Helicobacter pylori. Int $j$ Cancer. 2015;136:487-490. doi:10.1002/ijc.28999

23. Fang J-Y, Zheng S, Jiang B, et al. Consensus on the Prevention, Screening, Early Diagnosis and Treatment of Colorectal Tumors in China: Chinese Society of Gastroenterology, October 14-15, 2011, Shanghai, China. Gastrointest Tumors. 2014;1:53-75. doi:10.1159/ 000362585 .

24. Quaife S, Forbes LJ, Ramirez A, et al. Recognition of cancer warning signs and anticipated delay in help-seeking in a population sample of adults in the UK. $B r J$ Cancer. 2014;110:12-18. doi:10.1038/ bjc. 2013.684

25. Moutel G, Duchange N, Lièvre A, et al. Low participation in organized colorectal cancer screening in France: underlying ethical issues. Eur J Cancer Prevent. 2019;28:27-32. doi:10.1097/ CEJ.0000000000000417

26. Di L, Wu H, Zhu R, et al. Multi-disciplinary team for early gastric cancer diagnosis improves the detection rate of early gastric cancer. BMC Gastroenterol. 2017;17:147. doi:10.1186/s12876-017-0711-9

\section{Publish your work in this journal}

Cancer Management and Research is an international, peer-reviewed open access journal focusing on cancer research and the optimal use of preventative and integrated treatment interventions to achieve improved outcomes, enhanced survival and quality of life for the cancer patient.
The manuscript management system is completely online and includes a very quick and fair peer-review system, which is all easy to use. Visit http://www.dovepress.com/testimonials.php to read real quotes from published authors. 